\title{
Transthyretin-Related Amyloidoses: A Structural and Thermodynamic Approach
}

\author{
Estefania Azevedo, Priscila F. Silva, \\ Fernando Palhano, Carolina A. Braga and \\ Debora Foguel
}

Additional information is available at the end of the chapter

http://dx.doi.org/10.5772/53148

\section{Introduction}

The amyloidoses comprise a spectrum of diseases caused by the systemic or localised deposition of characteristic fibrillar material, termed amyloid fibrils [1]. These deposits can be found in various organs and tissues throughout the body [1]. Each amyloidosis is classified according to the chemical nature of the protein that forms the initial amyloid fibril deposit (Table 1). Amyloid fibrils are ubiquitous structures that are rich in cross $\beta$-sheets and typically have a fibrillar morphology, which can vary in length and diameter [1]. Amyloid fibrils are detected in vitro and in vivo using specific-binding molecules, namely Congo Red [1], thiophene derivatives [2] and Thioflavin-S and T [1]. The most common amyloidoses are Alzheimer's and Parkinson's disease and type 2 diabetes, in which amyloid fibrils are found deposited in the central nervous system and in beta cells from the pancreas, respectively [1]. This chapter will cover the transthyretin (TTR)-related amyloidoses, a group of diseases that roughly affects approximately 8,000-10,000 people worldwide [3]. These amyloidoses are caused by the aggregation of TTR, an amyloidogenic protein that can give rise to amyloid fibrils [4].

Transthyretin (TTR) was first discovered in the cerebrospinal fluid (CSF) in $1942[5,6]$ and then sequenced in 1984 [7], receiving the name prealbumin because of its electrophoretic migration pattern compared to albumin. Afterwards, aiming to better describe its functionality, its name was changed to transthyretin- the transporter of thyroxine (T4) and retinol [5]. TTR transports retinol through binding to retinol-binding protein (RBP) and T4 due to the formation of a hydrophobic channel, which exists only when TTR is tetrameric (Figure 1). Al- 
though its function may vary, TTR is highly conserved from humans to bacteria [8]. TTR is a $55 \mathrm{kDa}$ homotetrameric protein predominantly synthesised by the liver and choroid plexus, which is located in the brain. Although TTR is known primarily as a transporter, emerging evidence has demonstrated that TTR can also act as a protease [9] and a neuroprotective molecule [10].

\begin{tabular}{|c|c|c|}
\hline Amyloidoses & Amyloidogenic Protein & Tissues Affected \\
\hline Alzheimer's Disease & Amyloid- $\beta$ & Brain \\
\hline Parkinson's Disease & $\alpha$-synuclein & Brain \\
\hline Primary Systemic Amyloidosis & Immunoglobulin heavy or light chain & $\begin{array}{l}\text { Mesenchymal tissue, tongue, heart, } \\
\text { skin, gastrointestinal tract }\end{array}$ \\
\hline $\begin{array}{l}\text { Dialysis-related } \beta 2 \text {-microglobulin } \\
\text { Amyloidosis }\end{array}$ & $\beta 2$-microglobulin & Kidney \\
\hline Senile Systemic Amyloidosis & Wild-type TRR & Heart \\
\hline Familial Amyloid Cardiomyopathy & Mutated TTR & Heart \\
\hline Familial Amyloid Polyneuropathy & Mutated TTR & Peripheral nervous system \\
\hline $\begin{array}{l}\text { Central Nervous System-associated } \\
\text { Amyloidosis Or Oculoleptomeningeal } \\
\text { Amyloidosis }\end{array}$ & Mutated TTR & Brain, Meninges \\
\hline $\begin{array}{l}\text { Hereditary Fibrinogen Alpha-chain } \\
\text { Renal Amyloidoses }\end{array}$ & Fibrinogen alpha-chain & Kidney \\
\hline Apolipoprotein A Amyloidosis & Apolipoprotein A-I, A-II and A-III & Kidney, liver, heart \\
\hline $\begin{array}{l}\text { Hereditary Cistatin CAmyloid } \\
\text { Angiopathy }\end{array}$ & Cistatin C & Brain \\
\hline Gelsolin Amyloidosis & Gelsolin & $\begin{array}{l}\text { Peripheral nervous system } \\
\text { Heart }\end{array}$ \\
\hline $\begin{array}{l}\text { Familial Renal Amyloidosis Or } \\
\text { Lysozyme Amyloidosis }\end{array}$ & Lysozyme & Kidney \\
\hline $\begin{array}{l}\text { Cerebral Amyloid Angiopathy British- } \\
\text { type }\end{array}$ & Abri & Brain \\
\hline $\begin{array}{l}\text { Cerebral Amyloid Angiopathy Danish- } \\
\text { type }\end{array}$ & Adan & Brain \\
\hline Spongiform Encephalopathy & Cellular prion protein (Scrapie form) & Brain \\
\hline Medullary Thyroid Carcinoma & Calcitonin & Thyroid \\
\hline Type 2 Diabetes & Islet amyloid polypeptide (amylin) & Pancreas \\
\hline Atrial Amyloidosis & Atrial natriuretic peptide & Heart \\
\hline Age-related Pituitary Amyloidosis & Prolactin & Pituitary \\
\hline Insulinomes & Insulin & $\begin{array}{l}\text { Pancreas or insulinome-associated } \\
\text { tissue }\end{array}$ \\
\hline Aortic Medial Amyloidosis & Lactadherin & Heart \\
\hline Hereditary Lattice Corneal Dytrophy & keratoepithelin & Cornea \\
\hline $\begin{array}{l}\text { Familial Supepithelial Corneal } \\
\text { Amyloidosis }\end{array}$ & Lactoferrin & Cornea \\
\hline $\begin{array}{l}\text { Ameloblast-associated Odontogenic } \\
\text { Tumour }\end{array}$ & Odontogenic protein & Mouth \\
\hline Senile Seminal Amyloidosis & Semenogelin & Seminal Gland \\
\hline $\begin{array}{l}\text { Tauopathies associated with ageing or } \\
\text { secondary to Alzheimer's Disease }\end{array}$ & Tau & Brain \\
\hline Huntington's Disease & Hungtintin & Brain \\
\hline Familial Encephalopathy & Neuroserpin & Brain \\
\hline
\end{tabular}

Table 1. Amyloidogenic proteins and tissues affected in human amyloidoses. Adapted from Hamilton and Benson, 2001. 
The crystallographic structure of human TTR, which was solved in 1971 [11], revealed that each TTR monomer is composed of 127 amino acid residues, forming $8 \beta$-strands named from $\mathrm{A}-\mathrm{H}$, which are arranged in a $\beta$-sandwich of two four-stranded $\beta$-sheets and one small $\alpha$-helix found between $\beta$-strands E and F [11, 12]. TTR monomers interact via hydrogen bonds between the antiparallel, adjacent $\beta$-strands $\mathrm{H}_{-} \mathrm{H}^{\prime}$ and $\mathrm{F}-\mathrm{F}^{\prime}$ to form a dimeric species. The two dimers (A-B and C-D) predominantly form the tetramer through hydrophobic contacts between the residues of the A-B and G-H loops. The tetramer forms a central hydrophobic pocket (T4 channel) with two binding sites for hormones [11, 12]. Each TTR monomer contains one cysteine residue at position 10 and two tryptophan residues at positions 41 and 79, which can be used as a tool for monitoring TTR unfolding [13]. Many groups have studied the structure of TTR and its aggregation process to understand the triggering factors that favour TTR aggregation, which occurs in a nonnucleated manner that is known as a downhill polymerisation reaction because tetramer dissociation into monomers is the rate-liming step of the aggregation reaction [14]. Based on this model, many studies have focused on developing effective and selective TTR ligands that can prevent TTR dissociation and aggregation [15].

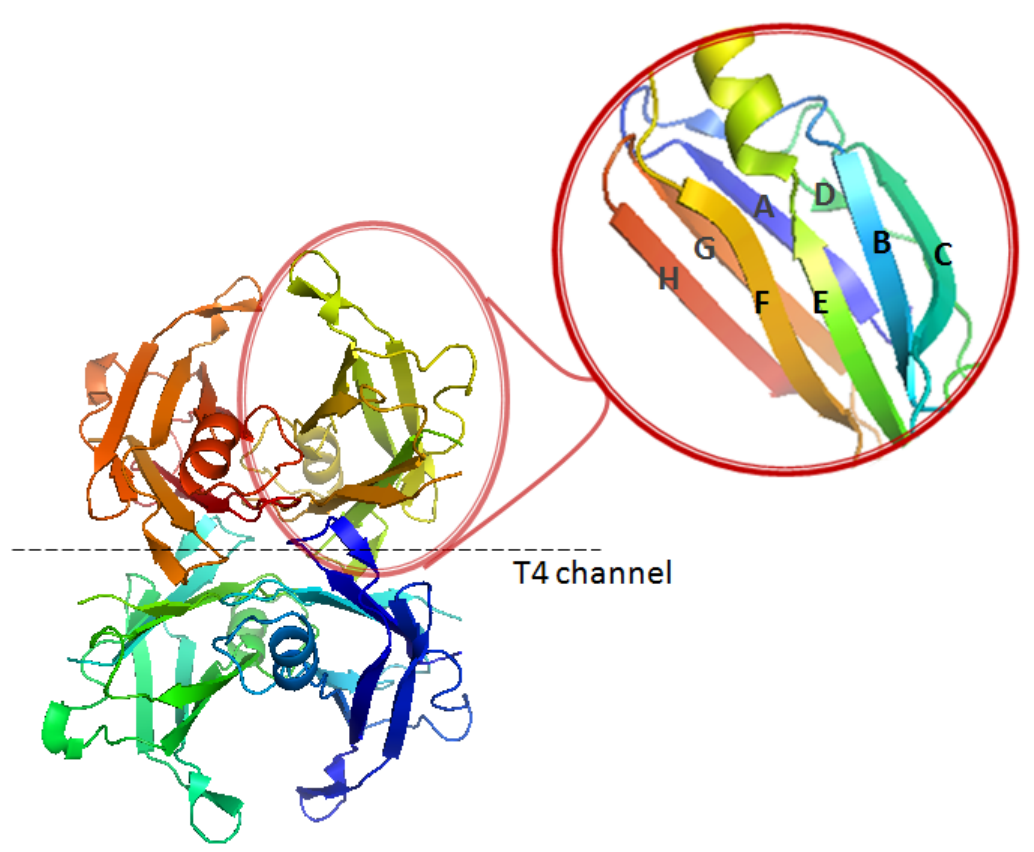

Figure 1. X-ray structure of Transthyretin. TTR is a homotetrameric protein composed of four monomers of 127 amino acids. Structurally, in its native state, TTR contains eight stands $(\mathrm{A}-\mathrm{H})$ and a small $\mathrm{a}$-helix. The contacts between the dimers form two hydrophobic pockets where T4 binds (T4 channel). As shown in red, each monomer contains one small $a$-helix and eight $\beta$-strands (CBEF and DAGH). Adapted from a model; PDB code 1DVQ. 
Thus, in the present review, we aim to appraise the literature related to TTR aggregation and focus on the contribution of the thermodynamics and structural aspects related to tetramer dissociation and monomer unfolding, which appears to be the basis for TTR amyloid formation.

\section{TTR-related amyloidoses}

The TTR gene, which is located on chromosome 18 at position 12.1 [16], presents many polymorphisms, except for one deletion, leading to over 80 amino acid substitutions in the TTR polypeptide sequence [16]. Many TTR variants, such as V30M and L55P, are associated with familial amyloidotic polyneuropathy (FAP), a lethal autosomal dominant disorder in which mutant forms of TTR aggregate to form amyloid fibrils that deposit in tissues, especially the peripheral nervous system (PNS). These amyloid deposits are predominantly composed of intact or fragmented TTR in the form of fibrillar species that progressively accumulate [16, 17]. The initial symptom is typically a sensory peripheral neuropathy in the lower limbs, with pain and severely affected temperature sensation and later followed by motor impairments [17]. Most patients with FAP have early and severe impairment of the autonomic nervous system, commonly manifested by dyshidrosis, sexual impotence, alternating diarrhoea and constipation, orthostatic hypotension, and urinary incontinence [17-19]. The disease onset typically depends on the mutation and the ethnic background of each patient and begins at approximately the third or fourth decade of life, although there are many cases in which the disease onset occurs later in life [17-19]. Not all TTR variant gene carriers develop FAP, indicating that other factors must be involved in the development of clinical symptoms [19].

Not only are most TTR mutants prone to aggregation, wild-type TTR (wt-TTR) possesses an inherent, although low, potential to undergo aggregation and form amyloid fibrils (predominantly in the heart). Hence, it is estimated that approximately $25 \%$ of the world population over 80 years old presents some cardiac amyloid deposits composed of wt-TTR [20]. This form of TTR amyloidosis is known as senile systemic amyloidosis (SSA). SSA patients typically present with congestive heart failure and arrhythmia [20,21]. Recently, it was demonstrated that wt-TTR can aggregate in many tendons and ligaments in aged individuals [22]. Interestingly, among all patients who exhibit SSA symptoms, the male gender predominates, suggesting that gender is a significant disease modifier [20, 22].

Some mutations of TTR, such as V122I, have also been associated with cardiac amyloidosis and are frequently found among African descendants, even though the age of onset varies significantly among patients [23]. This form of TTR-related amyloidosis is termed familial amyloid cardiomyopathy (FAC). FAC symptoms comprise an increase in ventricular wall thickness and an increase in parietal stiffness, which leads to a precipitous increase in ventricular pressure $[23,24]$.

Although rare, another form of TTR amyloidosis has been described as predominantly affecting the central nervous system and is, therefore, known as central nervous system amy- 
loidosis (CNSA) or oculoleptomeningeal amyloidosis (OA) [25]. Approximately 13 different mutations, such as D18G and A25T, have been associated with the development of CNSA in humans $[25,26]$. This form of amyloidosis is believed to occur due to the high instability of TTR mutants, leading to protein degradation by the endoplasmic reticulum-associated degradation (ERAD) pathway in hepatocytes and preferential secretion by the choroid plexus epithelium [27]. This preferential secretion by the choroid plexus is believed to be caused by a higher concentration of T4, which when bound to TTR stabilises the tetramer and favours its secretion by choroid plexus cells [27]. The age of onset is approximately the fourth to fifth decade of life, and amyloid accumulation typically occurs in brain vessels and leptomeninges, predominantly leading to subarachnoideal bleeding [25]. Some affected individuals may also develop hydrocephalus with increased CSF pressure and relatively high CSF protein levels [25]. Leptomeningeal involvement can extend down to the spinal cord, leading to symptoms related to spinal cord or spinal nerve compression [25]. Most patients have little, if any, systemic amyloid deposition. In addition to CNS deposits, TTR amyloid fibrils can also accumulate in the eye vitreous humour, leading to blindness [25].

\section{Therapies for TTR-related amyloidoses: When and where to inhibit aggregation?}

The treatment for TTR-related amyloidoses varies according to the symptoms presented by the patient (cardiac, autonomic or central). Currently, FAP is the only form of TTR-related amyloidoses that has a treatment, which is orthotopic liver transplantation (OLT) [28]. Although a treatment exists, some patients have displayed disease progression after OLT, which has been shown to be due to continued amyloid formation from wt-TTR and is especially true in patients with active FAC symptoms [28, 29]. In addition, after OLT, some patients develop CNSA symptoms [30,31] due to the production of mutant TTR by the choroid plexus. Another problem related to OLT therapy is that this procedure is also known as "domino liver transplantation", in which the liver of the FAP patient is transplanted into a non-FAP patient. However, the non-FAP liver receptors develop FAP symptoms in less than 5 years and not later in life as expected [32]. These phenomena suggest that OLT therapy is possible but that it is not a fully effective and safe procedure. In addition, for FAC or SSA patients, heart transplantations have only been performed successfully worldwide in a few patients presenting cardiac amyloidosis [33]. In summary, these clinical data emphasise the importance of developing new therapies for TTR-related amyloidosis.

\section{TTR-stabilisers: A new and powerful approach to stop TTR aggregation}

TTR can bind a variety of molecules, from small hydrophobic compounds to large glycosaminoglycans, with great flexibility [15]. As mentioned before, TTR is composed of four iden- 
tical monomeric subunits that assemble around a central channel; therefore, the tetramer possesses a molecular symmetry with two hormone-binding sites per tetramer (T4 channel). Under physiological conditions, only one of the hormone-binding sites of the T4 channel is occupied by T4 [34]. However, hormone binding is governed by a phenomenon termed negative cooperativity, in which the binding of T4 to the second binding site reduces the hormone-binding affinity of the first hormone-binding site $[15,34]$. The fact that TTR is not the only T4 transporter in the human body [34] and that unbound T4 concentrations in blood are very low (less than $0.1 \mu \mathrm{M}, 34$ ) agree with the fact that most TTR in vivo does not have $\mathrm{T} 4$ bound to its two binding sites. This information has made it possible to design a novel therapy using small molecules that bind to the T4 channel with high affinity and reduce TTR aggregation.

Miroy et al. have shown that the binding of T4 itself stabilises the TTR tetramer and consequently inhibits amyloid formation [35]. Since then, many pharmacological agents and natural products, such as plant flavonoids, nonsteroidal anti-inflammatory drugs (NSAIDs), and inotropic bipyridines, have been tested, and these agents have been demonstrated to be strong competitors for T4 binding to TTR and even possess higher binding affinities than T4 [15, 36, 37]. Interestingly, many of these small molecules share structural similarities with T4, typically presenting one or more aromatic rings. X-ray crystallographic studies have demonstrated that these compounds bind to the TTR hydrophobic channel, similarly to T4, due to the hydrophobic properties of the compounds [15, 36]. As observed for T4 and even RBP, as mentioned above, the binding of NSAIDs to TTR is typically negatively cooperative. However, depending on the ligand, this cooperativity may change. The currently tested NSAIDS include diclofenac, diflunisal and flufenamic acid [15, 36-39]. Interestingly, the first two compounds are currently approved by the Food and Drug Administration (FDA) for the treatment of other pathological conditions. Structural data for TTR complexed with flufenamic acid and diflunisal demonstrate that these compounds mediate hydrophobic and hydrogen bond interactions between the subunits, which stabilise tetrameric TTR [36, 38, 39]. The detailed comparisons of several structures of apo TTR (native TTR) and TTR complexes with small molecules provide insights into the mechanism of ligand-induced conformational changes on ligand binding [40, 41]. Although promising, the chronic use of NSAIDS has been correlated with dyspepsia symptoms, small intestine bleeding and ulcers [42].

Natural polyphenols (curcumin and epigallocatechin gallate) and flavonoids (quercetin and chrisin) have also been successful in inhibiting wt- and V30M TTR aggregation under acidic conditions $[43,44]$. Genistein, an isoflavone compound, is also able to inhibit TTR fibril formation by stabilising the native tetramer [45]. Interestingly, both flavonoids and genistein interact with Lys15, Ser117 and Thr119, enhancing tetramer stability [44, 45] and implicating these amino acids in preventing TTR aggregation. In addition, a TTR point mutation (Thr119Met) has been demonstrated to be highly stable to denaturising agents [46], corroborating the idea that the amino acids around the hormone-binding sites aide in tetramer stabilisation. 
Recently, a new TTR-stabilising drug, Tafamidis, was approved for use in Europe. This class of compounds functions by preventing the formation of amyloid fibrils, retaining the native tetrameric state of TTR $[15,47,48]$. This mode of action is possible because the most accepted theory regarding amyloid fibril formation is that TTR tetramers need to dissociate to a partially folded monomer, which then rapidly self-assembles to give rise to soluble oligomer intermediates before reaching the amyloid state [12].

There are many studies introducing the use of TTR-stabilising drugs or antisense oligonucleotides (ASO) as new, more effective therapies against TTR amyloidosis. There is currently an on-going trial to test the efficacy of using ASO specifically to target TTR in FAP patients and its safety in healthy subjects [ISIS pharmaceuticals, ISI-TTR ${ }_{\mathrm{rx}}$ 49]. The rationale behind ASO therapy and other small interfering RNA (siRNA) therapies [49-51] is that these oligonucleotides interfere with TTR production in the liver and choroid plexus, inhibiting its production and, therefore, its deposition as amyloid fibrils [49, 50,52]. Data have shown that RNA interference techniques have been successful; however, this technique may still be unsuccessful for dealing with old, existing amyloid deposits.

As mentioned above, the use of various compounds as TTR-stabilisers relies on the hypothesis that TTR needs to dissociate to form fibrils (Figure 2). Although there is evidence supporting this hypothesis, our group has shown that a cycle of compression-decompression using high hydrostatic pressure (HHP) is able to produce an altered wt-TTR species (T4*) that remains tetrameric and that undergoes aggregation under mild acidic conditions $(\mathrm{pH}$ 5-5.6), where untreated wt-TTR remains soluble [53] (Figure 2).

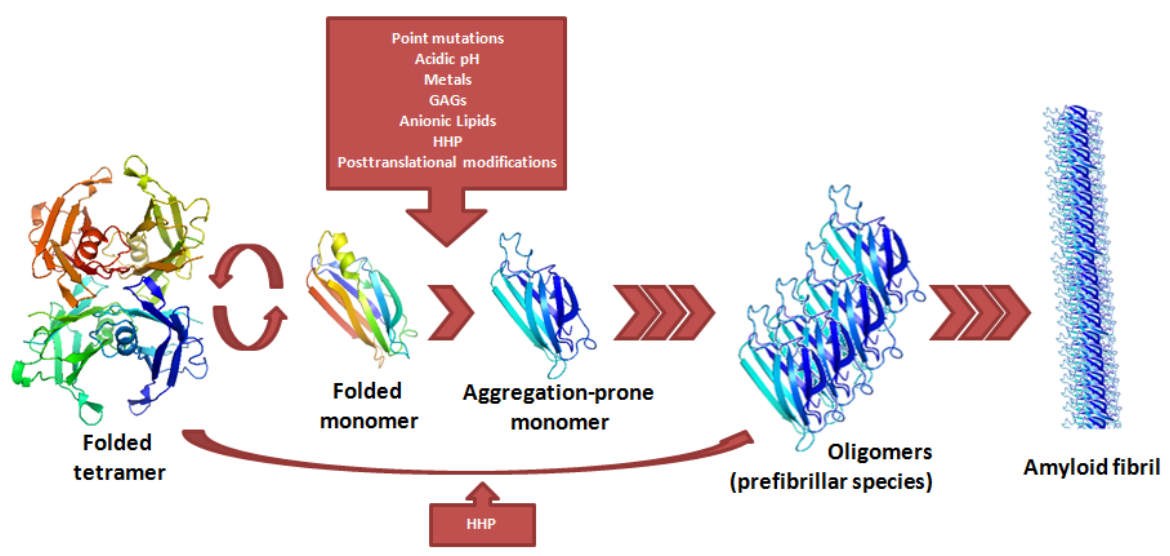

Figure 2. Transthyretin (TTR) amyloid cascade. TTR maintains a tetramer to monomer dissociation rate, normally forming folded monomers. These monomers can partially unfold due to point mutations, acidic conditions, metals, glycosaminoglycans (GAGs), anionic lipids, high hydrostatic pressure (HHP) and posttranslational modifications. This unfolded monomer is prone to aggregation, forming prefibrillar species such as oligomers and, consequently, mature amyloid fibrils. In addition, the alternatively folded tetramers formed during a compression-decompression cycle of HHP can aggregate either directly or after dissociating into monomers. 
An alternative way to approach this problem is to design drugs that disrupt existing amyloid fibrils. To this end, another class of molecules known as tetracyclines, such as doxycycline, is able to interfere with TTR fibrillogenesis in vitro [54], disrupting TTR fibrils. Interestingly, these molecules do not act as inhibitors of TTR fibril formation; however, these molecules disrupt fibrils [54]. The in vivo use of doxycycline in a murine FAP model exhibits beneficial effects [54], suggesting that this alternative approach may be as effective as the use of TTR stabilisers. However, it is unclear whether the products of fibril breakdown are toxic. The anthracycline compound described by Palha et al., which is known as 4 '-deoxi-4'iododoxorubicina (IDOX) and which has been used in the treatment of patients diagnosed with monoclonal immunoglobulin light chain (AL) amyloidosis [55], is able to efficiently disrupt TTR amyloid fibrils into amorphous aggregates [56]. Although IDOX displayed promising effects, it was later demonstrated that IDOX is cardiotoxic and is not indicated for use for that reason [57]. However, this new class of compounds has provided a new way of understanding TTR pathogenesis and helped to design new and clinically safer fibril-disrupting drugs.

\section{Why TTR aggregates?}

Although restricted to a single amino acid substitution, TTR mutations may significantly alter the kinetics of TTR aggregation [27]. In trying to understand which features of TTR mutants are responsible for its altered amyloidogenicity, a large amount of crystallographic data has been collected by different groups worldwide. Unfortunately, the conclusion reached was that there was no significant deviation in TTR structure. The only exception to this conclusion was that there were significant deviations for the positions at which the amino acid substitutions took place [58]. However, these single deviations were not significant enough to account for the variability in TTR mutation aggressiveness. Although TTR mutations do not significantly alter the tetrameric structure compared to wt-TTR [58], a single amino acid substitution is enough to alter the thermodynamic stability and rate of dissociation of the tetramer [27]. Many in vitro studies using chaotropic agents such as urea or guanidine chloride and HHP have revealed that thermodynamic stability and the rate of tetramer dissociation links certain TTR mutations to variant amyloidogenicity. In one study, Sekijima et al. conducted a global energetic analysis of 23 disease-associated TTR mutants, observing that the rate of tetramer dissociation and the thermodynamic stability in increasing concentrations of urea predicted the aggressiveness of the mutants [27]. Our group has collected supporting data concerning the latter hypothesis that the amyloidogenic mutants A25T and L55P, which exhibit an aggressive phenotype, have lower thermodynamic stabilities than wt-TTR $[59,60]$. In addition, we have demonstrated that other amyloidogenic mutants with exacerbated profiles of aggregation and with high tetramer dissociation rates, when HHP is applied, are typically associated with the development of clinically severe FAP [60]. These studies have been corroborated by Hammarstrom et al. [46], who observed that one particular TTR mutation, T119M, which is clinically associated with a phenomenon known as trans-suppression, dissociated at a 40 -fold slower rate than wt-TTR. These dissoci- 
ation rates allow this mutant to be significantly more stable than any of the other described mutants and highly resistant to high urea concentrations for long periods of time [27, 46]. Indeed, this mutation clinically induces trans-suppression, a phenomenon where a patient bearing the V30M mutation, an amyloidogenic variant associated with FAP, along with the T119M mutation does not develop amyloid deposits and, therefore, has no FAP symptoms [46]. In fact, it was later demonstrated that the TTR tetramers that circulated in this patient's bloodstream were hybrids composed of T119M and V30M monomers [46]. These hybrid tetramers gained the characteristic stability of $\mathrm{T} 119 \mathrm{M}$, which overcame the characteristic instability of V30M, and these properties allowed the hybrid tetramers to remain intact and prevent the formation of amyloid fibrils [46].

In addition, the X-ray crystallographic data for the V30M variant, which is also involved in FAP, revealed a perturbation of the $\beta$-sheets, which led to a distortion of the T4-binding channel that weakens the hormone interactions [61]. The X-ray structure of another highly amyloidogenic mutant, known as L55P, revealed a disruption of the hydrogen bonds between strands D and A, which produces different interface contacts and, in contrast to what has been mentioned previously, results in eight monomers that have different packing arrangements than that described for wt-TTR [62]. In addition, the highly amyloidogenic, yet engineered, triple mutant G53S/E54D/L55S reveals a new conformation with a novel $\beta$-slip conformation, which results in the shift of three residues in strand D and places Leu58 at the position occupied by Leu55. This new conformation impacts the binding interactions with retinol-binding protein [63]. Together, these data lead us to believe that mutations that influence the unfolding of monomers create species that are prone to aggregation. The contribution of the inserted amino acid does not alter the overall structure of the protein but may be capable of interfering with the aggressiveness of the disease, of impacting tetramer stability and dissociation rates and of directing the aggregate to specific tissues such as the peripheral nerves, heart, and meninges.

Although these structural alterations may predict TTR amyloidogenicity, some studies have also shown that because of the presence of cysteine residues (4 per tetramer), TTR is more prone to be posttranslationally modified, such as by S-thiolation and S-sulphonation [64, 65], which have been shown to affect TTR amyloidogenicity. In addition, only 5-15\% of circulating TTR is free of posttranslational modifications [66]. One study has also shown that the interaction of TTR with anionic lipids and cholesterol might accelerate the aggregation process [67].

TTR aggregation, especially in vivo, does not occur without additional proteins. In fact, in addition to the factors mentioned above, there are many other factors involved in TTR aggregation. The TTR amyloid fibrils from FAP patient deposits and nearly all amyloid fibrils in vivo are found to be co-aggregated with many other molecules such as serum amyloid $\mathrm{P}$ (SAP), heparan sulphate, and metalloproteinases, which makes it even more difficult to analyse which one of these molecules actually contributes to TTR aggregation and clearance in vivo and in vitro [1, 68-70]. Among the various molecules that co-aggregate with TTR, glycosaminoglycans (GAGs) are able to accelerate the aggregation of TTR in vitro, and this phenomenon is dependent on the degree of GAG sulphation [69]. Because the GAG composition and concentrations vary among different tissues, this fact might partly explain 
the specificity of these aggregates for certain tissues [69]. Murakami et al. has described the effect of augmented levels of SAP in the deposition of TTR fibrils in the tissue of TTR transgenic mice [70]. Although SAP was found to be associated with TTR deposits, their findings suggest that SAP does not affect the severity or onset of TTR deposition into the gastrointestinal tract of mice [70]. Our group has recently shown that the TTR fibrils formed from a CNSA-related mutant, A25T, are able to sequester a diversity of molecules from human CSF during aggregation [59]. Many of these molecules are associated with inflammation and coagulation, suggesting that in vivo these pathways may also contribute to TTR-related amyloidosis. Indeed, in many of the amyloidotic tissues from FAP patients, an upregulation of inflammation-associated molecules such as inducible nitric oxide synthase (iNOS), IL-1 $\beta$ and TNF- $\alpha$ [68] has been found. Interestingly, a report by Saito et al. showed that nitric oxide (NO) has an important role in TTR amyloid formation because of the S-nitrosylation of cysteine 10 of TTR monomers [71].

TTR aggregation can also be triggered by mild acidification ( $\mathrm{pH}$ 5.0-4.0) [73], cold storage [74] and the presence of metals such as $\mathrm{Zn}^{2+}[72,75]$. Using X-ray crystallography, Palaninathan et al. observed that acidic conditions increase the probability that the EF helix-loop of the B monomer of TTR changes its conformation, leading to destabilisation of the TTR tetramer and favouring TTR aggregation [73]. In addition, upon further acidification $(<\mathrm{pH}$ 3.9), TTR monomers can adopt a different conformation that may not be in the classic folding pathway of TTR but is able to aggregate without reaching the amyloid fibril [73]. Olofsson et al. have shown that prior to aggregation, the first step in TTR destabilisation occurs in the C-D $\beta$-strands, which dislocate when exposed to acidic conditions [76]. More data have shown that not only are the C-D $\beta$-strands more susceptible to destabilisation but also the entire $\beta$-sheet sandwich CBEF actually dislocates upon acidification [77], suggesting that this region of TTR may be responsible, in part, for the first structural changes preceding TTR dissociation. Recently, Bateman et al. demonstrated that wt-TTR fibril cores were predominantly composed of the C-terminal region and described a different pathway of aggregation that may not require loosening of the above-mentioned TTR regions [78]. Interestingly, the majority of amyloidogenic mutations, which are associated with FAP, FAC or OA, are found within the $\beta$-strands of the CBEF $\beta$-sheet sandwich [77]. Other groups also believe that acidic conditions may make an important contribution to TTR aggregation both in vitro and in vivo, in which the amyloid process may take place inside endosomes and lysosomes $[79,80]$.

Previous data have shown that high concentrations of metals, such as $\mathrm{Zn}^{2+}$ and $\mathrm{Cu}^{2+}$, can induce TTR amyloid formation in vitro [81]. Additionally, chelating agents, such as EDTA or EGTA, are able to disrupt amyloid fibrils, suggesting that metals affect the stability of TTR fibrils [81]. Interestingly, $\mathrm{Zn}^{2+}$ was also found in ex vivo ocular amyloid deposits from FAP patients, indicating the possible role of metals in the pathogenesis of FAP [82]. Our group has shown that increasing concentrations of $\mathrm{Zn}^{2+}$ are able to perturb, predominantly under acidic conditions, the loop EF helix loop, a region involved in RBP binding [75]. This region has recently been implicated in this phenomenon by two other groups, as the X-ray structures of wt-TTR and two variants, I84A and I84S, demonstrated a structural perturbation in the EF helix loop under acidic conditions [83]. 


\section{Conclusion}

Although many studies have attempted to infer the conditions that affect TTR stability and amyloidogenicity, why the disease penetrance, pathology and clinical course are so different between mutations is still not completely understood. Herein, we present some of the data obtained by different groups that provides evidence on TTR stability and amyloidogenicity. Many groups have collected data on TTR stability by studying the structure of TTR and designing TTR-binding compounds. However, as further questions have been raised about TTR stability, more evidence has demonstrated that most of the answers with regard to TTR stability lie within its thermodynamic properties and rate of tetramer dissociation.

\section{Acknowledgements}

We would like to thank the funding agencies: Conselho Nacional de Desenvolvimento Científico e Tecnológico (CNPq), Coordenação de Aperfeiçoamento de Pessoal de Nível Superior (CAPES) and Fundação de Amparo a Pesquisa Carlos Chagas Filho do Estado do Rio de Janeiro (FAPERJ).

\section{Author details}

Estefania Azevedo ${ }^{1}$, Priscila F. Silva ${ }^{1}$, Fernando Palhano ${ }^{1,2}$, Carolina A. Braga ${ }^{1,3}$ and Debora Foguel ${ }^{1}$

1 Instituto de Bioquimica Medica, Universidade Federal do Rio de Janeiro, Rio de Janeiro, Brazil

2 The Scripps Research Institute, La Jolla, California, USA

3 Polo de Xerem, Universidade Federal do Rio de Janeiro, Duque de Caxias, Brazil

\section{References}

[1] Chiti F, Dobson CM. Protein misfolding, functional amyloid, and human disease. Annual Review of Biochemistry 2006; 75, 333-366.

[2] Aslund A, Sigurdson CJ, Klingstedt T, Grathwohl S, Bolmont T, Dickstein DL, Glimsdal E, Prokop S, Lindgren M, Konradsson P, Holtzman DM, Hof PR, Heppner FL, Gandy S, Jucker M, Aguzzi A, Hammarström P, Nilsson KP. Novel pentameric thio- 
phene derivatives for in vitro and in vivo optical imaging of a plethora of protein aggregates in cerebral amyloidoses. ACS Chem Biol. 2009; 4(8):673-84.

[3] Benson MD, Kincaid JC.The molecular biology and clinical features of amyloid neuropathy. Muscle Nerve. 2007; 36(4):411-23.

[4] Serpell LC, Sunde M, Fraser PE, Luther PK, Morris EP, Sangren O, Lundgren E, Blake CC. Examination of the structure of the transthyretin amyloid fibril by image reconstruction from electron micrographs. Journal of Molecular Biology. 1995; 254(2): $113-8$.

[5] Hamilton JA, Benson MD. Transthyretin: a review from a structural perspective.Cellular and Molecular Life Sciences. 2001; 58(10):1491-521.

[6] Kabat EA, Moore DH, Landow H. An electrophoretic study of the protein components in cerebrospinal fluid and their relationship to the serum proteins. Journal of Clinical Investigation. 1942; 21(5):571-7

[7] Mita S, Maeda S, Shimada K, Araki S. Cloning and sequence analysis of cDNA for human prealbumin. Biochemica et Biophysica Research Communications. 1984; 124(2):558-64.

[8] Cendron L, Ramazzina I, Percudani R, Rasore C, Zanotti G, Berni R.Probing the evolution of hydroxyisourate hydrolase into transthyretin through active-site redesign. Journal of Molecular Biology. 2011; 409(4):504-12.

[9] Liz MA, Faro CJ, Saraiva MJ, Sousa MM. Transthyretin, a new cryptic protease. Journal of Biological Chemistry. 2004; 279(20):21431-8.

[10] Said G. Familial amyloid polyneuropathy: mechanisms leading to nerve degeneration. Amyloid. 2003; 1:7-12.

[11] Blake CC, Swan ID, Rerat C, Berthou J, Laurent A, Rerat B. An x-ray study of the subunit structure of prealbumin. Journal of Molecular Biology. 1971; 61(1):217-24

[12] Foss TR, Wiseman RL, Kelly JW. The pathway by which the tetrameric protein transthyretin dissociates. Biochemistry. 2005; 44(47):15525-33.

[13] Silva JL, Cordeiro Y, Foguel D. Protein folding and aggregation: two sides of the same coin in the condensation of proteins revealed by pressure studies. Biochimica et Biophysica Acta. 2006; 1764(3):443-51.

[14] Hurshman AR, White JT, Powers ET, Kelly JW. Transthyretin aggregation under partially denaturing conditions is a downhill polymerization. Biochemistry. 2004;43(23): 7365-81.

[15] Johnson SM, Connelly S, Fearns C, Powers ET, Kelly JW..The transthyretin amyloidoses: from delineating the molecular mechanism of aggregation linked to pathology to a regulatory-agency-approved drug. Journal of Molecular Biology. 2012;421(2-3): 185-203. 
[16] Saraiva MJ. Transthyretin mutations in health and disease. Human Mutations. 1995;5(3):191-6.

[17] Ando Y, Suhr OB.Autonomic dysfunction in familial amyloidotic polyneuropathy (FAP). Amyloid. 1998; 5(4):288-300.

[18] Alves IL, Altland K, Almeida MR, Winter P, Saraiva MJ. Screening and biochemical characterization of transthyretin variants in the Portuguese population. Human $\mathrm{Mu}$ tations. 1997;9(3):226-33.

[19] Tanaka M, Hirai S, Matsubara E, Okamoto K, Morimatsu M, Nakazato M. Familial amyloidotic polyneuropathy without familial occurrence: carrier detection by the radioimmunoassay of variant transthyretin. Journal of Neurology, Neurosurgery and Psychiatry. 1988; 51(4):576-8.

[20] Westermark P, Sletten K, Johansson B, Cornwell GG 3rd. Fibril in senile systemic amyloidosis is derived from normal transthyretin. Proccedings in National Academy of Sciences of United States of America. 1990; 87(7):2843-5.

[21] Sekijima Y, Kelly JW, Ikeda S. Pathogenesis of and therapeutic strategies to ameliorate the transthyretin amyloidoses. Current Pharmaceuthical Design. 2008;14(30): 3219-30.

[22] Sueyoshi T, Ueda M, Jono H, Irie H, Sei A, Ide J, Ando Y, Mizuta H.Wild-type transthyretin-derived amyloidosis in various ligaments and tendons. Human Pathology. 2011;42(9):1259-64.

[23] Rapezzi C, Quarta CC, Riva L, Longhi S, Gallelli I, Lorenzini M, Ciliberti P, Biagini E, Salvi F, Branzi A.Transthyretin-related amyloidoses and the heart: a clinical overview. Nature Review Cardiology. 2010;7(7):398-408.

[24] Dungu JN, Anderson LJ, Whelan CJ, Hawkins PN.Cardiac transthyretin amyloidosis. Heart. 2012 Aug 11.

[25] Benson MD. Leptomeningeal amyloid and variant transthyretins. American Journal of Pathology. 1996;148(2):351-4.

[26] Liepnieks JJ, Dickson DW, Benson MD. A new transthyretin mutation associated with leptomeningeal amyloidosis. Amyloid. 2011; 18 Suppl 1:155-7.

[27] Sekijima Y, Wiseman RL, Matteson J, Hammarström P, Miller SR, Sawkar AR, Balch WE, Kelly JW.The biological and chemical basis for tissue-selective amyloid disease. Cell. 2005;121(1):73-85

[28] Hund E, Linke RP, Willig F, Grau A.Transthyretin-associated neuropathic amyloidosis. Pathogenesis and treatment.Neurology. 2001;56(4):431-5.

[29] Liepnieks JJ, Benson MD.Progression of cardiac amyloid deposition in hereditary transthyretin amyloidosis patients after liver transplantation. Amyloid. 2007;14(4): 277-82. 
[30] Liepnieks JJ, Zhang LQ, Benson MD. Progression of transthyretin amyloid neuropathy after liver transplantation. Neurology. 2010; 75(4):324-7.

[31] Munar-Qués M, Salva-Ladaria L, Mulet-Perera P, Solé M, López-Andreu FR, Saraiva MJ.Vitreous amyloidosis after liver transplantation in patients with familial amyloid polyneuropathy: ocular synthesis of mutant transthyretin.Amyloid. 2000;7(4):266-9.

[32] Takei Y, Gono T, Yazaki M, Ikeda S, Ikegami T, Hashikura Y, Miyagawa S, Hoshii Y. Transthyretin-derived amyloid deposition on the gastric mucosa in domino recipients of familial amyloid polyneuropathy liver. Liver Transplantation 2007; 13, 21518.

[33] Ammirati E, Marziliano N, Vittori C, Pedrotti P, Bramerio MA, Motta V, Orsini F, Veronese S, Merlini PA, Martinelli L, Frigerio M.The first Caucasian patient with p.Val122Ile mutated-transthyretin cardiac amyloidosis treated with isolated heart transplantation.Amyloid. 2012;19(2):113-7.

[34] Bartalena, L. \& Robbins, J. (1993). Thyroid hormone transport proteins. Clin. Lab. Med. 13, 583-598.

[35] Miroy GJ, Lai Z, Lashuel HA, Peterson SA, Strang C, Kelly JW.Inhibiting transthyretin amyloid fibril formation via protein stabilization. Procedings of the National Academy of Sciences of the United States of America. 1996;93(26):15051-6

[36] Cody V.Mechanisms of molecular recognition: crystal structure analysis of human and rat transthyretin inhibitor complexes. Clinical Chemistry and Laboratory Medicine. 2002;40(12):1237-43.

[37] Trivella DB, Sairre MI, Foguel D, Lima LM, Polikarpov I.The binding of synthetic triiodo 1-thyronine analogs to human transthyretin: molecular basis of cooperative and non-cooperative ligand recognition. Journal of Structural Biology. 2011;173(2): 323-32

[38] Adamski-Werner SL Palaninathan SK, Sacchettini,JC, Kelly J W. Diflunisal analogues stabilize the native state of transthyretin. Potent inhibition of amyloidogenesis. Journal of Medical Chemistry.2004; 47,355-374.

[39] Baures PW, Oza VB, Peterson SA, Kelly JW. Synthesis and evaluation of inhibitors of transthyretin amyloid formation based on the non-steroidal anti-inflammatory drug, flufenamic acid. Bioorganic and Medical Chemistry.1999; 7, 1339-1347.

[40] Almeida MR, Gales L, Damas AM, Cardoso I, Saraiva MJ.Small transthyretin (TTR) ligands as possible therapeutic agents in TTR amyloidoses. Current Drug Targets: CNS and Neurological Disorders. 2005; 4(5):587-96

[41] Neumann P, Cody V, Wojtczak A.Structural basis of negative cooperativity in transthyretin. Acta Biochimica Polonica. 2001; 48(4):867-75. 
[42] Graham DY, Opekun AR, Willingham FF, Qureshi WA.Visible small-intestinal mucosal injury in chronic NSAID users. Clinical Gastroenterology and Hepatology. 2005; 3(1):55-9.

[43] Ferreira N, Saraiva MJ, Almeida MR. Natural polyphenols inhibit different steps of the process of transthyretin (TTR) amyloid fibril formation. FEBS Letters. 2011; 585(15):2424-30.

[44] Trivella DB, Dos Reis CV, Lima LM, Foguel D, Polikarpov I.Flavonoid interactions with human transthyretin: Combined structural and thermodynamic analysis. Journal of Structural Biology. 2012; in press.

[45] Trivella DB, Bleicher L, Palmieri Lde C, Wiggers HJ, Montanari CA, Kelly JW, Lima LM, Foguel D, Polikarpov I. Conformational differences between the wild type and V30M mutant transthyretin modulate its binding to genistein: implications to tetramer stability and ligand-binding. Journal of Structural Biology. 2010; 170(3):522-31.

[46] Hammarström P, Schneider F, Kelly JW. Trans-suppression of misfolding in an amyloid disease. Science. 2001; 293(5539):2459-62.

[47] Bulawa CE, Connelly S, Devit M, Wang L, Weigel C, Fleming JA, Packman J, Powers ET, Wiseman RL, Foss TR, Wilson IA, Kelly JW, Labaudinière R. Tafamidis, a potent and selective transthyretin kinetic stabilizer that inhibits the amyloid cascade. Proccedings of the National Academy of Sciences of the Unites States of America. 2012;109(24):9629-34.

[48] Coelho T, Maia LF, Martins da Silva A, Waddington Cruz M, Planté-Bordeneuve V, Lozeron P, Suhr OB, Campistol JM, Conceição IM, Schmidt HH, Trigo P, Kelly JW, Labaudinière R, Chan J, Packman J, Wilson A, Grogan DR.Tafamidis for transthyretin familial amyloid polyneuropathy: A randomized, controlled trial. Neurology. 2012

[49] Benson MD, Pandey S, Witchell D, Jazayeri A, Siwkowski A, Monia B, Kluve-Beckerman B. Antisense oligonucleotide therapy for TTR amyloidosis.Amyloid. 2011; 18; $1-55$.

[50] Ackermann EJ, Guo S, Booten S, Alvarado L, Benson M, Hughes S, Monia BP.Clinical development of an antisense therapy for the treatment of transthyretin-associated polyneuropathy. Amyloid. 2012; 1:43-4.

[51] Hayashi Y, Mori Y, Higashi T, Motoyama K, Jono H, Sah DW, Ando Y, Arima H. Systemic delivery of transthyretin siRNA mediated by lactosylated dendrimer $/ \alpha$-cyclodextrin conjugates into hepatocyte for familial amyloidotic polyneuropathy therapy.Amyloid. 2012; 1:47-9.

[52] Benson MD, Smith RA, Hung G, Kluve-Beckerman B, Showalter AD, Sloop KW, Monia BP.Suppression of choroid plexus transthyretin levels by antisense oligonucleotide treatment. Amyloid. 2010;17(2):43-9. 
[53] Ferrão-Gonzales AD, Souto SO, Silva JL, Foguel D.The preaggregated state of an amyloidogenic protein: hydrostatic pressure converts native transthyretin into the amyloidogenic state. Proccedings of the National Academy of Sciences of the United States of America. 2000;97(12):6445-50.

[54] Cardoso I, Saraiva MJ.Doxycycline disrupts transthyretin amyloid: evidence from studies in a FAP transgenic mice model. FASEB Journal. 2006; 20(2):234-9.

[55] Gertz MA, Lacy MQ, Dispenzieri A, Cheson BD, Barlogie B, Kyle RA, Palladini G, Geyer SM, Merlini G. A multicenter phase II trial of 4'-iodo-4'deoxydoxorubicin (IDOX) in primary amyloidosis (AL). Amyloid. 2002; 9(1):24-30.

[56] Merlini G, Ascari E, Amboldi N, Bellotti V, Arbustini E, Perfetti V, Ferrari M, Zorzoli I, Marinone MG, Garini P, et al. Interaction of the anthracycline 4'-iodo-4'-deoxydoxorubicin with amyloid fibrils: inhibition of amyloidogenesis. Proccedings of the National Academy of Sciences of the United States of America. 1995; 92(7):2959-63.4-9.

[57] Danesi R, Bernardini N, Agen C, Costa M, Macchiarini P, Della Torre P, Del Tacca M.Cardiotoxicity and cytotoxicity of the anthracycline analog 4'-deoxy-4'-iodo-doxorubicin.Toxicology. 1991;70(2):243-53.

[58] Hörnberg A, Eneqvist T, Olofsson A, Lundgren E, Sauer-Eriksson AE.A comparative analysis of 23 structures of the amyloidogenic protein transthyretin. Journal of Molecular Biology. 2000;302(3):649-69.

[59] Azevedo EP, Pereira HM, Garratt RC, Kelly JW, Foguel D, Palhano FL. Dissecting the structure, thermodynamic stability, and aggregation properties of the A25T transthyretin (A25T-TTR) variant involved in leptomeningeal amyloidosis: identifying protein partners that co-aggregate during A25T-TTR fibrillogenesis in cerebrospinal fluid. Biochemistry. 2011; 50(51):11070-83.

[60] Ferrão-Gonzales AD, Palmieri L, Valory M, Silva JL, Lashuel H, Kelly JW, Foguel D.Hydration and packing are crucial to amyloidogenesis as revealed by pressure studies on transthyretin variants that either protect or worsen amyloid disease. Journal of Molecular Biology. 2003;328(4):963-74.

[61] Hamilton JA, Steinrauf LK, Braden BC, Liepnieks J, Benson MD, Holmgren G, Sandgren $\mathrm{O}$, Steen L. The x-ray crystal structure refinements of normal human transthyretin and the amyloidogenic Val-30-->Met variant to 1.7-A resolution. Journal of Biological Chemistry. 1993; 268(4):2416-24.

[62] Sebastião MP, Saraiva MJ, Damas AM. The crystal structure of amyloidogenic Leu55 --> Pro transthyretin variant reveals a possible pathway for transthyretin polymerization into amyloid fibrils.Journal of Biological Chemistry. 1998; 273(38):24715-22.

[63] Karlsson A, Sauer-Eriksson AE. Heating of proteins as a means of improving crystallization: a successful case study on a highly amyloidogenic triple mutant of human 
transthyretin. Acta Crystallographica Section F: Structural Biology and Crystallization Communications. 2007; 63(Pt 8):695-700.

[64] Nakanishi T, Yoshioka M, Moriuchi K, Yamamoto D, Tsuji M, Takubo T. S-sulfonation of transthyretin is an important trigger step in the formation of transthyretin-related amyloid fibril. Biochimica et Biophysica Acta. 2010 ;1804(7):1449-56

[65] Lim A, Prokaeva T, McComb ME, Connors LH, Skinner M, Costello CE.Identification of S-sulfonation and S-thiolation of a novel transthyretin Phe33Cys variant from a patient diagnosed with familial transthyretin amyloidosis. Protein Science. 2003; 12(8):1775-85.

[66] Hagen GA, Elliott WJ. Transport of thyroid hormones in serum and cerebrospinal fluid.The Journal of Clinical Endocrinology and Metabolism. 1973; 37(3):415-22.

[67] Hou X, Mechler A, Martin LL, Aguilar MI, Small DH. Cholesterol and anionic phospholipids increase the binding of amyloidogenic transthyretin to lipid membranes. Biochimica et Biophysica Acta. 2008; 1778(1):198-205.

[68] Cardoso I, Brito M, Saraiva MJ.Extracellular matrix markers for disease progression and follow-up of therapies in familial amyloid polyneuropathy V30M TTR-related. Disease Markers. 2008; 25(1):37

[69] Bourgault S, Solomon JP, Reixach N, Kelly JW.Sulfated glycosaminoglycans accelerate transthyretin amyloidogenesis by quaternary structural conversion.Biochemistry. 2011; 15;50(6):1001-15.

[70] Murakami T, Yi S, Maeda S, Tashiro F, Yamamura K, Takahashi K, Shimada K, Araki S.Effect of serum amyloid P component level on transthyretin-derived amyloid deposition in a transgenic mouse model of familial amyloidotic polyneuropathy. American Journal of Pathology. 1992; 141(2):451-6.

[71] Saito S, Ando Y, Nakamura M, Ueda M, Kim J, Ishima Y, Akaike T, Otagiri M.Effect of nitric oxide in amyloid fibril formation on transthyretin-related amyloidosis. Biochemistry. 2005; 44(33):11122-9.

[72] Bonifácio MJ, Sakaki Y, Saraiva MJ. 'In vitro' amyloid fibril formation from transthyretin: the influence of ions and the amyloidogenicity of TTR variants. Biochimica et Biophysica Acta. 1996; 1316(1):35-42

[73] Palaninathan SK, Mohamedmohaideen NN, Snee WC, Kelly JW, Sacchettini JC.Structural insight into $\mathrm{pH}$-induced conformational changes within the native human transthyretin tetramer. Journal of Molecular Biology. 2008; 382(5):1157-67.

[74] Sörgjerd K, Klingstedt T, Lindgren M, Kågedal K, Hammarström P.Prefibrillar transthyretin oligomers and cold stored native tetrameric transthyretin are cytotoxic in cell culture. Biochemical and Biophysical Research Communications. 2008; 377(4): $1072-8$ 
[75] Palmieri Lde C, Lima LM, Freire JB, Bleicher L, Polikarpov I, Almeida FC, Foguel D. Novel Zn2+-binding sites in human transthyretin: implications for amyloidogenesis and retinol-binding protein recognition. Journal of Biological Chemistry. 2010; 285(41):31731-41

[76] Olofsson A, Ippel JH, Wijmenga SS, Lundgren E, Ohman A.Probing solvent accessibility of transthyretin amyloid by solution NMR spectroscopy. Journal of Biological Chemistry. 2004; 279(7):5699-707.

[77] Liu K, Cho HS, Lashuel HA, Kelly JW, Wemmer DE.A glimpse of a possible amyloidogenic intermediate of transthyretin. Nature Structural Biology. 2000 ;7(9):754-7.

[78] Bateman DA, Tycko R, Wickner RB. Experimentally derived structural constraints for amyloid fibrils of wild-type transthyretin. Biophysical Journal. 2011; 101(10):2485-92.

[79] Chang MH, Hua CT, Isaac EL, Litjens T, Hodge G, Karageorgos LE, Meikle PJ.Transthyretin interacts with the lysosome-associated membrane protein (LAMP-1) in circulation. Biochem J. 2004; 382(Pt 2):481-9.

[80] Purkey HE, Dorrell MI, Kelly JW. Evaluating the binding selectivity of transthyretin amyloid fibril inhibitors in blood plasma. Proccedings of the National Academy of Sciences of United States of America. 2001; 98(10):5566-71.

[81] Wilkinson-White LE, Easterbrook-Smith SB. Characterization of the binding of Cu(II) and $\mathrm{Zn}(\mathrm{II})$ to transthyretin: effects on amyloid formation. Biochemistry. 2007; 46(31): 9123-32.

[82] Susuki S, Ando Y, Sato T, Nishiyama M, Miyata M, Suico MA, Shuto T, Kai H.Multielemental analysis of serum and amyloid fibrils in familial amyloid polyneuropathy patients. Amyloid. 2008; 15(2):108-16.

[83] Pasquato N, Berni R, Folli C, Alfieri B, Cendron L, Zanotti G. Acidic pH-induced conformational changes in amyloidogenic mutant transthyretin. Journal of Molecular Biology. 2007; 366(3):711-9 